\title{
jReality, jtem, and oorange - a way to do math with computers
}

\author{
Tim Hoffmann ${ }^{1}$ and Markus Schmies ${ }^{2}$ \\ 1 Mathematisches Institut der Universität München \\ Theresienstr. 39, D-80333 München, Germany \\ hoffmann@mathematik . uni-muenchen. de \\ 2 Technische Universität Berlin \\ Straße des 17. Juni 136, D-10623 Berlin, Germany \\ schmies@math.tu-berlin.de
}

\begin{abstract}
This paper presents our approach to the question of how to code mathematics (mostly experimantal and motivated from geometry) in Java. We are especially interested in the question how the development of mathematical software and the mathematics itself influence each other and how the design of programming tools and code can support this interrelationship.
\end{abstract}

\section{1 introduction}

There are many ways computers are utilized to do mathematics. The area spreads from computer algebra and computer proofs to numerical analysis and scientific computing. For all of these applications there are powerfull tools available.

The software tools and design paradigms we want to present here can not be sorted easily into these categories:

Computer Algebra Systems (CAS) like MATHEMATICA [28], Maple [14], MuPad [20] or Gap, singular, and magma to name a few are all capable of powerfull symbolic manipulation and algebraic calculations. While there is a well working expression parser in the jtem project symbolic calculations are not the main focus of our system. Our components are designed to support a special way of software/application development which is not present in CAS systems, although they (usually) have powerfull programing languages built in. On the other hand one can not say that our development environment oorange is an integrated development environment (IDE) in the classical sense (like e.g. eclipse [6] or JBuilder for Java): It has 
for example no debugger but allows changes to a running program and it uses a graphical interface for the control flow (see section 4). Here GRAPE [9] shows the object oriented approach while AVS [3] has a similar graphical interface.

There is some similarity to dynamic geometry software like Cinderella [24, 25], Cabri [5], or geometer's sketchpad [7] (again the list is not complete) in the sense that both types of systems can be used in a similar way: to investigate geometric facts, examples, and hypotheses by interactively changing configurations or "parameters". But even if there is a $2 \mathrm{~d}$ viewer in jtem we are more concerned with $3 \mathrm{~d}$ graphics than $2 \mathrm{~d}$ which is the classical domain of the dynamic geometry (there is a $3 \mathrm{~d}$ version of Cabri though) and do not relay on projective geometry and we do no automated prooving (like e.g. Cinderella).

This is again a complete field on its own (see for example the theorema project [13] which is build on top of MATHEMATICA)

Of course there are many well established systems and viewers for 3d graphics available (vtk [27], open scene graph [21], open inventor [22], aviatrix3d [2] and many more), and some of them are readily tailored for mathematical applications (like geomview [8] or javaview [10] and in some sense the 3DXplorMath [1]) but all of them missed at least one of the requirements we had for a viewer suited for our workflow ansatz (lacking e.g. thread saveness or hardware independence or hardware acceleration - see section 3).

The projects done in the spirit of our approach include numerically demanding ones (like [26] where workstation clusters were used for the search for heliciods with handles) but again while providing a set of standard algorithms for basic tasks we do not claim to be as effective or complete as many of the well established libraries and tools for numerical analysis or scientific computing [29,30]. This is traditionally an area where FORTRAN language shows its strength.

Our software and workflow arose from the needs and experiences our group has made in the last twenty years and the problems or tasks adressed by them origin mainly in discrete and differential geometry. Still they have a wide variety reaching from theory of discrete curves and surfaces, numerics on Riemann surfaces, and surface theory and their visualization. In these areas "mathematical computer 
experiments" became a common research tool early on, due to the visual character of the field (see e. g. [16]).

Mathematical computer experiments and programming mathematical concepts often do not follow the classical software engineering paradigms of modelling, specification, coding, and testing, but follow rather some concepts of extreme programming [4]. In particular setting up a mathematical experiment usually involves trying various approaches while inspecting the data and data flow closely. In short: these experiments often don't converge to a stable application that is ready for release. Instead the first results most likely give rise to new ideas of needs for modifications which hopefully will give new insight and so on. In these cases one can not any longer distinguish between the phases of development and usage of the software.

Another crucial factor is the ease of maintenance and deployment. Our experience is that in the long run it is not feasible to maintain and deploy software experiments with complicated library dependencies (after all math departments are no software development companies and do not have the manpower to support such developments). In addition it becomes more difficult for other people to run or verify the experiments. Whether an experiment is set up for teaching or for research purposes only, it seems most desirable to have the option of publishing it on the web. These general considerations already give rise to the following design paradigms:

1. maintainability

2. easy deployment and interoperability

3. adaption to mathematical work flow

The following sections feature our approach to a framework for the development of mathematical software which tries to address several of the above mentioned needs. In section 2 we discuss our observations of how experiments and applications grow and evolve and what is needed to support this life-cycle. After a short overview of the tools and packages we developed in section 3, section 4 presents an example that shows how our tools can be used to build fully functional applications. 


\section{2 our design decisions}

The design decisions presented here are driven by the experiences we gained from previous work. From 1994 to 1997 our group developed a prototyping environment for experimental mathematics: Oorange (Object ORiented Analysis Numerics and Graphics Environment) [15]. The Oorange project had a hybrid language scheme: numerics and graphics were implemented in Objective-C; the graphical programming environment used $\mathrm{Tcl}$ for scripting purposes. This decision was one of the reasons why the project finally came to the edge of maintainability. However in this process our design paradigms and a particular work flow emerged.

The first observation to make is that mathematical programming is neither data nor algorithm centered. This has several consequences: Firstly in an adapted programming environment both algorithms and (potentially rather complex) mathematical data objects need to be easily inspected and modified. Coding well defined tasks (like writing a library for a well understood piece of mathematics) is usually best done in a modern IDE like eclipse. But when doing an experiment we often come to a point where the current state might not be easily reproducible but one needs or wants to change the things one can do next in the program (this can be either changing or adding algorithms or equally important doing some visualization of the state). The paradigm for a design honoring this is: "Changing the code while keeping the data (or state) alive"

Secondly one can divide the algorithms in experiments roughly into two types: something one might call "fundamental" or "library" algorithms that are usually well understood and best placed in their own class or framework and "control flow" or "experimental" algorithms that one wants to change modify and adapt repeatedly. Of course there is no easy distinction between the two types. As an example for the first kind one might take minimizers: If you are not in the stage of developing a particular implementation, you usually use them as a black box: you will either change some parameters of the minimizer or replace it with another already tested algorithm. An example for the second kind could be the following scenario: To find the fix-points of a generic Möbius transformation one can pick an algorithm from a numerics package to find the Eigenvectors. This 
might be unstable. But since the fix-points are generically either attractive or repelling one can replace finding the Eigenvectors with iterating the Möbius transformation a couple of times. This is not something one wants to create a separate package for but rather just do it "on the spot". Thus a design should separate library and experimental code.

Thirdly the (mathematical) objects should be prepared to work in a large variety of algorithm frameworks (maybe from different origin) without modification. Usually adapting the objects to as many frameworks as possible is not feasible as it makes the classes less maintainable, more difficult to understand, and adds a lot of mutual dependencies to the packages. So the "gluing code" should be placed outside the objects and if possible the creation of the glueing code should be deferred to the moment a particular interface is needed.

When writing code, reusability and maintainability are prominent goals, since the publication of code can be viewed as a scientific goal. Using only one language greatly simplifies maintainability. However, writing experimental code stands against maintainability. Thus keeping the development of experimental code and application out of the library is essential for keeping the code of the libraries stable, clean, and orthogonal [18].

Finally one should mention that we learned that ideally library development and scripting style programming should be done in the same language.

\section{1 software life cycle}

As said above there is no strict border between library and experimental code. In our work flow code for problems that are well understood usually circulate in the group and become eventually (if general purpose enough) library code. But apart form that transition our experiments usually have the following life cycle.

1. create or gather classes or packages for "fundamental algorithms" and mathematical objects. Here "mathematical objects" can vary from complex numbers or quaternions to "a surface" and "fundamental algorithms" from say an ode solver to a special transformation of a surface. 
2. connect the algorithms and objects in an experimental algorithm and make it a running application with gui components

3 . inspect the result and modify the experimental algorithms repeatedly.

This is a kind of extreme or micro extreme programming, since the turnaround times are within hours not weeks.

\section{2 implications for the environment}

Modern CAS like MATHEMATICA, Maple or MuPad can in principle follow our work flow paradigms, but neither the interface of the IDE (notebook) nor the design of the languages are specifically adapted for this use: Notebook interfaces do not clearly show the dependencies of different parts of the code and for example rule based programming does not separate code and data at all. Some classical IDEs have code replacing features but this usually not intuitively usable to archive say a particular visualization.

Most modern scripting languages (including CAS systems) can interface external libraries but we learned that ideally library development and scripting style programming should be done in the same language. While IDEs support reusability and component based programming if the language does, giving access to all relevant data using the IDE should not give the feeling/speed of using a debugger.

Ideally the IDE should be free (or even better open source).

Our approach to address at least some of the shortcomings is oorange (see next section).

\section{3 implications for the programming language}

The projects presented here are developed using only pure Java as programming language (with the exception of jReality that has $o p$ tional support for OpenGL to have hardware accelerated 3d graphics if needed or wanted). Java offers mainstream solutions for all major technical problems we had to deal with in the original Oorange project. This enables us to focus on design, which we broke up into three separated projects: Java Oorange, jReality, and jtem. We will discuss them in greater detail in the next section. 
Besides of the modern design of the language Java has the advantage that the questions of easy deployment is virtually non existent anymore. The techniques of Java Webstart and Java applets allow to publish the finished experiments online.

Although the available code base is still one of the largest, FORTRAN never was an option for us since it is clearly not the first choice for graphics.

Using a CAS like MATHEMATICA or Maple as a language was no option either. There is virtually no easy way to do GUIs and although they have become quite performant already these languages have not the speed of Java or $\mathrm{C}++$. Moreover there is only minimal graphical interactivity without add ons and many of them are not free making deployment over the net unfeasible.

Said that, many of the presented design concepts are not really language dependent.

\section{3 a tour through the packages}

This section is not intended to be a tutorial or in depth description for any of the described packages but rather a short overview of some of the key concepts on which the packages are build.

jReality A scene graph library for 3d graphics [11]. While being focused purely on rendering and displaying 3d graphics, the library provides support for all stages and tasks in the visualization of experimental mathematics. It can do OpenGL accelerated rendering on workstations with suitable graphics cards, software only rendering for use in web presentations and applets, multi wall stereoscopic display on CAVE like environments with head tracking for immersive virtual reality, and output to renderman RIB or SVG or Postscript for high quality images for videos or publications.

It should be stressed that jReality is not able and not intended to do complex manipulations on the data or combinatorics of its input. Instead it provides convenient methods to set or update the data it displays and which is provided from somewhere else.

jReality strictly separates the scene graph it maintains from the displaying "back-end". This has some useful consequences. As an 
example, the tool system (the part responsible for rotating geometry, picking a point or in general user interaction with the scene) can not make any assumptions on the available input devices: While virtually every desktop computer has a mouse (which provides 2-3 (relative) degrees of freedom plus some buttons) there is no such thing in a CAVE like virtual environment here the user usually has a "wand" with 6 (absolute) degrees of freedom (3 for the position and 3 for the orientation of the device in space) plus some buttons. jReality abstracts from the actual device and instead maps the available freedoms to different transformation matrices which then will be used by the tools. Thus the same tool (like a rotate tool) will work in both setups without any changes.

jReality does not depend on jtem or oorange. For readers familiar with Java here is a code fragment that shows everything needed to open a frame that displays Enneper's minimal surface:

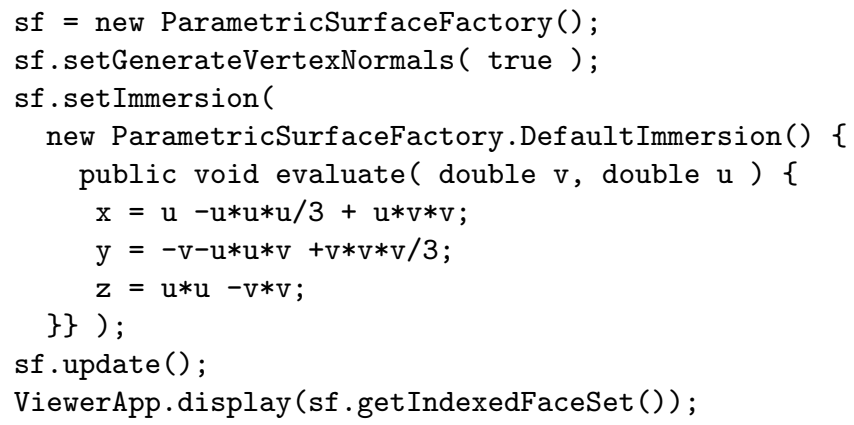

jReality will see its release summer 06 [12].

jtem (Java tools for experimental mathematics) The core of the jtem project $[17,26]$ consists of a collection of scientific, mostly mathematical, libraries implemented in pure standard Java2.

The project currently offers almost a dozen different subprojects, but its origin are three numerical libraries: numericalMethods, mfc, and riemann.

On a core level jtem provides numerical methods like minimizers and root finders, matrix factorization or algorithms for mesh generation. On a higher level there are classes that model complex numbers, quaternions or special matrix groups and basic linear algebra (blas). 
Here as well the idea of strict orthogonality is present since the dependencies are strictly one way: The high level classes will use the numerical methods, but the numerical methods can be used without any dependencies to the higher constructs. There is a package that provides elliptic functions and the library even has some gui components like a Möbius viewer (a $2 \mathrm{~d}$ viewer that has Möbius transformations a fundamental transformation group) or java2d (a Swing based $2 \mathrm{~d}$ viewer).

oorange The third component is more difficult to categorize: Oorange is a tool for rapid application development, that is especially designed for building applications (or experiments) while they run. The idea is that one can design the classes so that they model the mathematical objects they represent as closely as possible without the need to take care of interfaces or programming paradigms imposed by any other class or package that might be needed in a particular experiment. This interfacing (writing the glue) is done in and partially by - oorange.

Again oorange does not depend on any of the previous packages but will work with any Java code available.

In oorange the dependencies of the objects are represented as a directed graph with the objects sitting in the nodes. Objects are created by dragging them from a tree view of the Java classpath on a canvas. Changes in the state are propagated along the edges of the graph. The actions to be performed when a change is signaled is specified in the node's "brain".

The brain of a node provides methods that manage the interaction of the object with other objects. For each incoming edge the brain of a node has an (initially empty) method that is called whenever the source of that edge changes its state. One can fill these methods with any Java code needed to perform the appropriate action like setting some values on the object with data read from the object of the source node. The source code for these methods can be written and changed directly in oorange. It gets compiled and loaded on the fly.

Once a node is adapted to a special purpose it can be saved for later reuse. There is a large library of pre-made nodes for both math and gui building. 


\section{1 other packages we use}

Our goal of interoperability would be void if we were not able (or would not profit) from external packages. Among the ones we frequently use are: ant, antlr, xstream, batik, jogl, jinput, javaview, javahelp, beanshell, xpp, mtj, and many more. And of course we use IDEs like eclipse and the jdk from Sun.

\section{An example: geodesics on tori of revolution}

We want to illustrate the work flow by sketching the creation process of an application, which shows geodesics on tori of revolution. Markus has build, tested, and deployed it within a couple of hours on the occasion of a regular math course in 2003. It can be found in the virtual math labs at TU Berlin (www.math.tu-berlin.de/ geometrie/labs).

Even though the example is taken from an educational background it makes similar demands for an experimental environment than any of our typical research applications, e.g. complex user interface, visualization and graphical user interaction with two and three dimensional objects, computational intensive operations.

For such a setup it seems that major technical difficulties are inevitable, but we will show that in our environment these can be reduced to a minimum. This allows also the less experienced user to focus during the creation process of the application on the mathematics and still produces high performance code.

The path for creating the application is roughly as follows:

1. make an editable profile curve and display it.

One needs an periodic graphically editable profile curve. It should be $C^{2}$ since the geodesic equation is second order. Cubic b-splines fulfill all the requirements and are available in jtem. For editing we will use a java2d viewer component.

2. make a torus of revolution from the curve and display it.

We make the torus via parametric surface factory in jReality (we need to modify a node for that) and use an existing oorange node for the jReality viewer. 
3. generate and draw geodesics on the torus.

Adapt a node that utilize a numericalMethods ode solver to the geodesics equation, lift the result to the surface, and show it. It needs some more code and nodes, e.g. to edit the initial values and some parameters.

4. Finally make the application pretty.

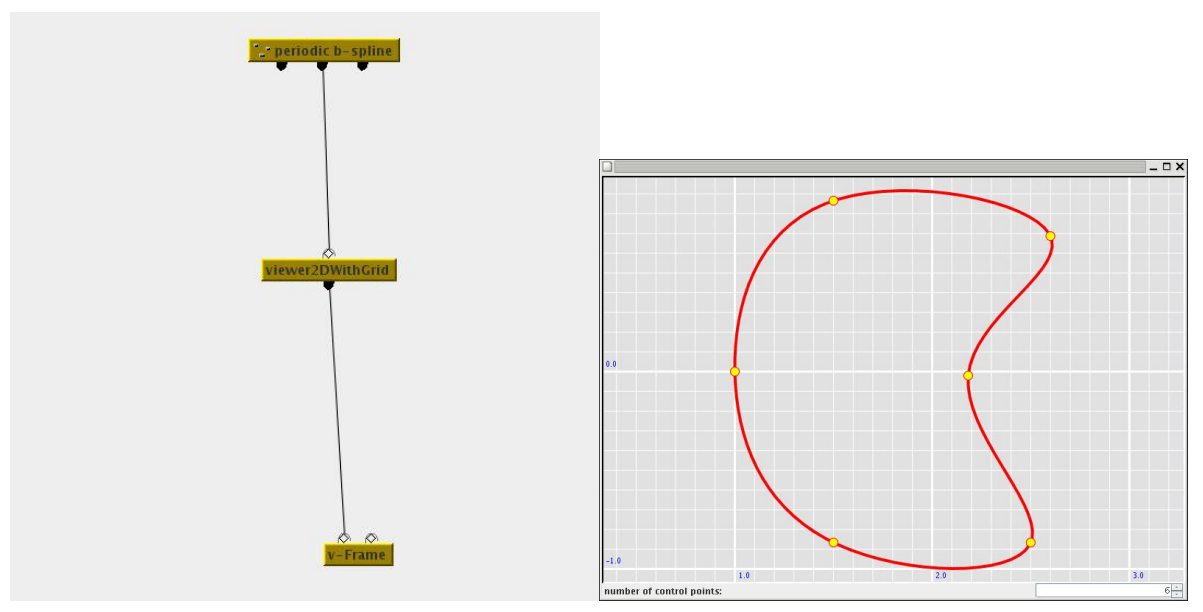

Fig. 1. oorange-network (left) and gui (right) of the application after Step 1

The fundamental numerical and visualization tasks of this example can be considered as typical for our group and are therefore covered by the libraries.

Planar curves appear in many of our applications: To get a graphical editable cubic b-spline curve one just needs to drag three existing oorange nodes into the oorange network editor and cable them. The top node is the actual b-spline. This node wraps the b-spline class available in jtem. The node in the middle is a viewer $2 \mathrm{~d}$ component that can display the spline. It gets the spline as an ingredient. Finally the $2 \mathrm{~d}$ viewer must be placed in a frame to show it. This is done by cabling it as an ingredient for a node that holds a Java frame [19]. After a couple of seconds without writing a single line of code we finished the first task (Figure 1). Already at this stage we have fully functional application with mathematical content and gui components, which allows us to check right away. 


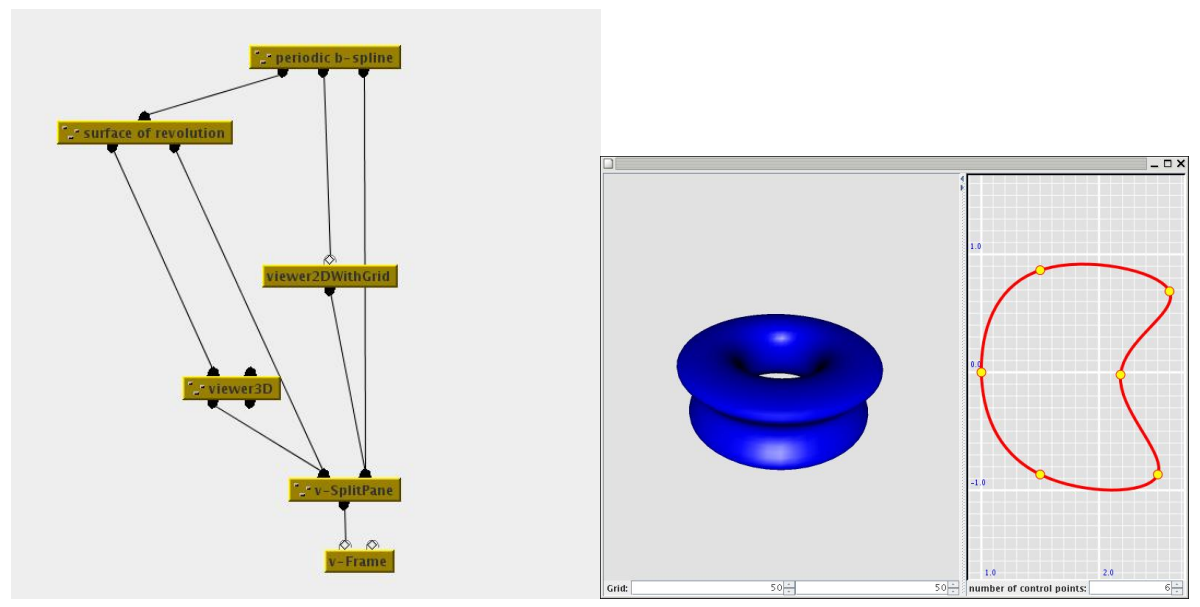

Fig. 2. oorange-network (left) and gui (right) of the application after Step 2

Also for a parametric surface there is a pre-made oorange node, which we have to adapt in order to get a surface of revolution. Therefore we cable the b-spline node with the parametric surface node; now we can adapt the formulas for the immersion referring to the b-spline. The lines to be adapted in the nodes brain are very similar to those presented as example jReality code on page 8:

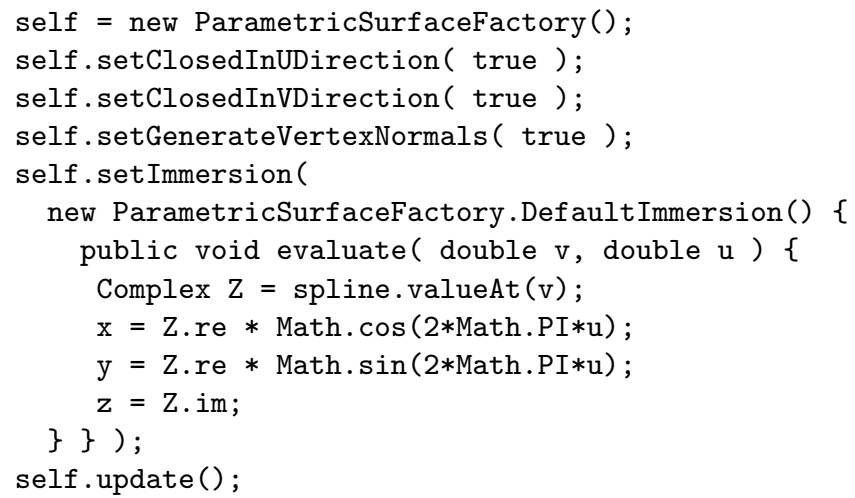

Now that the parametric surface is indeed a surface of revolution we rename the associated node. To visualize the surface we plug it into a $3 \mathrm{~d}$ viewer node, which we could pack above the $2 \mathrm{~d}$ viewer, showing the contour curve, by plugging it also into the frame. But the layout is nicer if we use a split pane [19]. The stage of the application is shown in Figure 2. 
Again we can check that everything works as intended. We have already a nice application just the geodesic is missing.

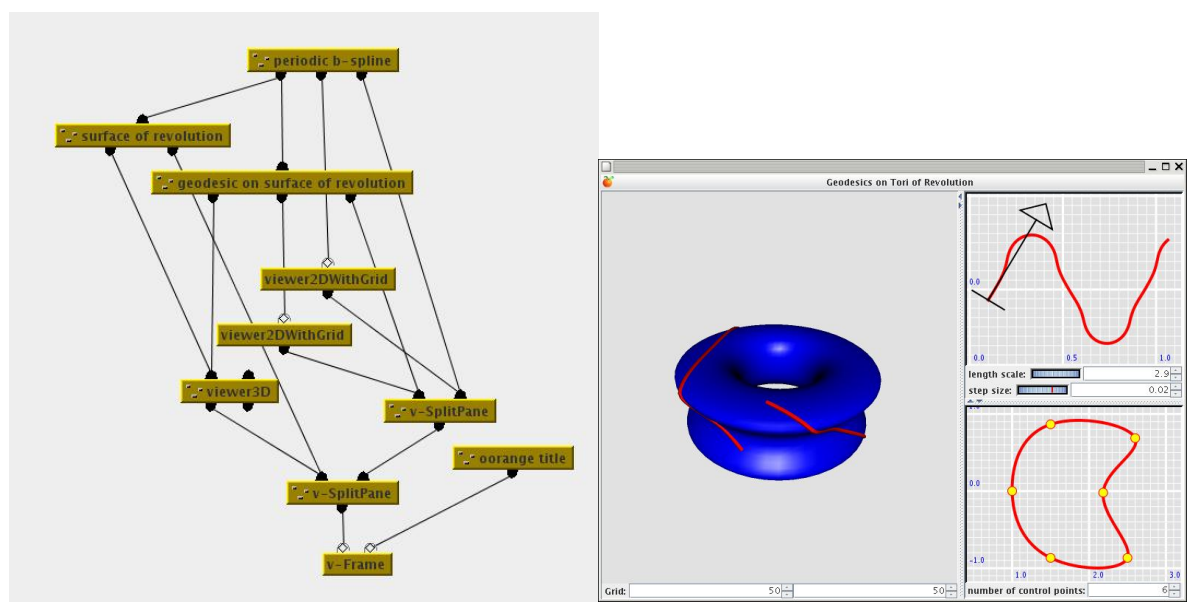

Fig. 3. oorange-network (left) and gui (right) of the application after Step 3

In the third step we adapt a general ode solver node that is based on a jtem solver to our needs. The numericalMethods solver needs an implementation of the interface "ODE" and the oorange node provides an empty implementation that needs to be filled. This is the implementation of the geodesic equation.

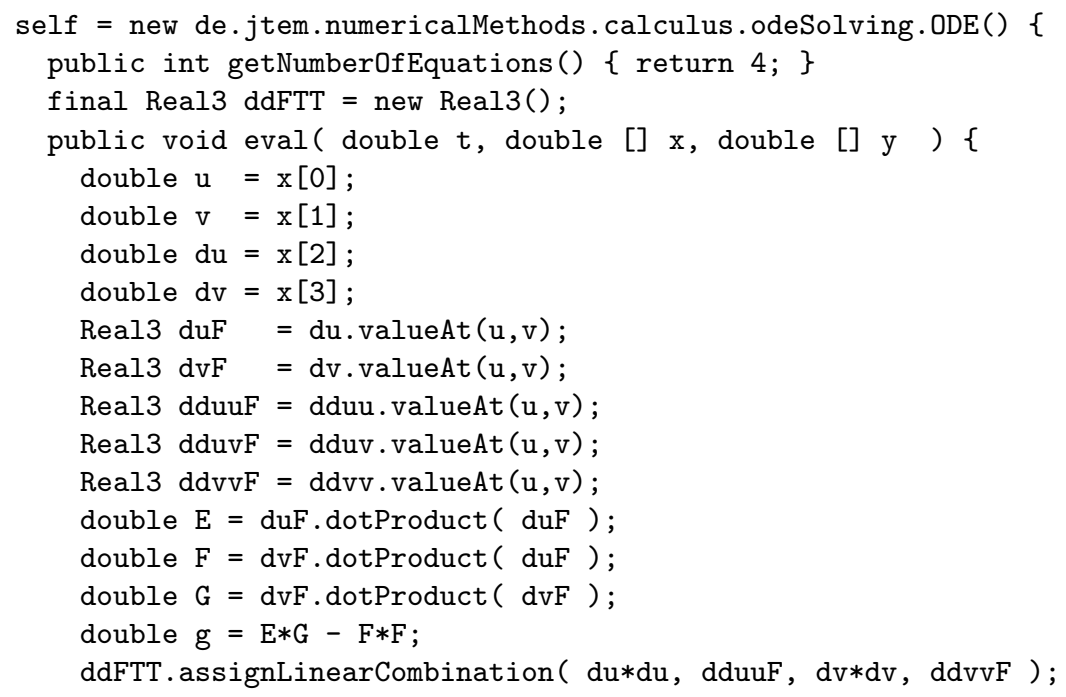




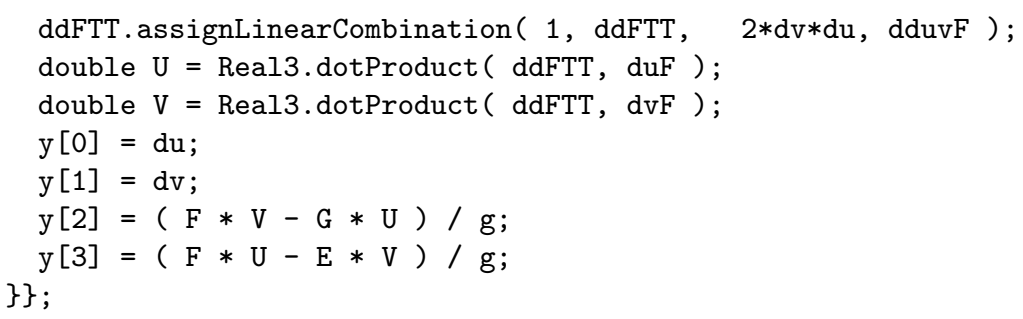

Entering the actual formulas that give the geodesic equation is obviously inevitable and the above code is just typical numerics code fragment in java which would look similar if it was written in say FORTRAN or C. But the important fact is here that this is by far the longest and the most complicated code which is needed for the whole application.

In the oorange network the component for the geodesic does a few more things. Besides the numerical part there is code that lifts the solution from the parameter domain to the surface, and there are gui components that allow the user to edit the initial values as well as to inspect the solution in the parameter domain. This component is visible in the above figure in the upper right. Figure 3 shows the final state of the network and the application as it was deployed (Java Webstart) for the math course.

The authors would like to thank Nadja Kutz for helpful discussions.

\section{References}

1. vmm.math.uci.edu/3D-XplorMath

2. aviatrix3d aviatrix3d.j3d.org

3. AVS www.avs.com/index_wf.html

4. K. Beck and C. Andres. extreme programming explained: embrace change, second edition, Addison-Wesley, ISBN 0321278658.

5. Cabri www-cabri.imag.fr/index-e.html

6. eclipse framework www.eclipse.org

7. geometer's sketchpad www.keypress.com/sketchpad

8. geomview www.geomview.org

9. GRAPE www.mathematik.uni-freiburg.de/IAM/Research/grape/GENERAL/

10. javaview ww. javaview.de

11. P. Brinkmann, Ch. Gunn, T. Hoffmann, H. Pietsch, M. Schmies, and S. Weißmann. www.jreality.de

12. P. Brinkmann, Ch. Gunn, T. Hoffmann, H. Pietsch, M. Schmies, and S. Weißmann. jReality - a thread-safe Java scene graph for mathematics. preprint, (2006), 10111018. 
13. B. Buchberger, C. Dupre, T. Jebelean, F. Kriftner, K. Nakagawa, D. Vasaru, and W. Windsteiger. The Theorema Project: A Progress Report. newblock In M. Kerber and M. Kohlhase, editors, Symbolic Computation and Automated Reasoning, pages 98-113, 2000. newblock www. theorema.org.

14. F. Gravan. the Maple book, CRCPress, 2001 newblock www .maplesoft.com.

15. C. Gunn, A. Ortmann, U. Pinkall, K. Polthier, and U. Schwarz. Oorange: A virtual laboratory for experimental mathematics. In H.-Chr. Hege and K. Polthier, editors, Visualization and Mathematics, 249-265, Springer, 1997.

16. A. J. Hanson, T. Munzner, and G. Francis. Interactive Methods for Visualizable Geometry IEEE Computer, Vol. 27, No. 4, pp. 73-83, 1994.

17. T. Hoffmann, S. Khadem, U. Pinkall, and M. Schmies. www.jtem.de

18. A. Hunt and D. Thomas. The Pragmatic Programmer: From Journeyman to Master. Addison-Wesley, Oct 1999. ISBN: 020161622X

19. javax.swing. JFrame and javax.swing. JSplitPane. java.sun.com/javase.

20. www.mupad.de

21. open scenegraph www.openscenegraph.org

22. open inventor www.tgs.com/

23. H. Pietsch, U. Pinkall and M. Schmies. www. oorange.de

24. J. Richter-Gebert and U. Kortenkamp. User Manual for the Interactive Geometry Software Cinderella Springer 2000, ISBN: 3-540-67139-0 www.cinderella.de

25. J. Richter-Gebert and U. Kortenkamp Euklidische und Nicht-Euklidische Geometrie in Cinderella. Journal für Mathematikdidaktik, 22:303-324, 2000.

26. M. Schmies. Computational Methods for Riemann Surfaces and Helicoids with Handles. PhD thesis, Technische Universität Berlin, 2005.

27. visualization toolkit public.kitware.com/VTK

28. St. Wolfram. the MATHEMATICA book, Wolfram Media/Cambridge University Press, 1999 www.wolfram.com.

29. LAPACK www.netlib.org/lapack

30. MATLAB www.mathworks.com 